\title{
Generation of obese rat model by transcription activator-like effector nucleases targeting the leptin receptor gene
}

\author{
Yuting $\mathrm{Chen}^{1 \dagger}$, Wenqing $\mathrm{Lu}^{1 \dagger}$, Na Gao ${ }^{1 \dagger}$, Yi Long ${ }^{1}$, Yanjiao Shao ${ }^{1}$, Meizhen Liu ${ }^{1}$, \\ Huaqing Chen ${ }^{1}$, Shixin $\mathrm{Ye}^{2}$, Xueyun $\mathrm{Ma}^{1}$, Mingyao Liu ${ }^{1,3^{*}} \&$ Dali $\mathrm{Li}^{1 * *}$ \\ ${ }^{1}$ Shanghai Key Laboratory of Regulatory Biology, Institute of Biomedical Sciences and School of Life Sciences, East China Normal University, \\ Shanghai 200241, China; \\ ${ }^{2}$ Ecole Normale Supérieure, Institut de Biologie de l' Ecole Normale Supérieure, Institut National de la Santé et de la Recherche Médicale \\ U1024, Centre National de la Recherche Scientifique, Unité Mixte de Recherche 8197, Paris F-75005, France; \\ ${ }_{3}^{3}$ Institute of Biosciences and Technology, Department of Molecular and Cellular Medicine, Texas A\&M University Health Science Center, \\ Houston, Texas 77030, USA
}

Received September 12, accepted November 6, 2015; published online May 25, 2016

\begin{abstract}
The laboratory rat is a valuable mammalian model organism for basic research and drug discovery. Here we demonstrate an efficient methodology by applying transcription activator-like effector nucleases (TALENs) technology to generate Leptin receptor (Lepr) knockout rats on the Sprague Dawley (SD) genetic background. Through direct injection of in vitro transcribed mRNA of TALEN pairs into SD rat zygotes, somatic mutations were induced in two of three resulting pups. One of the founders carrying bi-allelic mutation exhibited early onset of obesity and infertility. The other founder carried a chimeric mutation which was efficiently transmitted to the progenies. Through phenotyping of the resulting three lines of rats bearing distinct mutations in the Lepr locus, we found that the strains with a frame-shifted or premature stop codon mutation led to obesity and metabolic disorders. However, no obvious defect was observed in a strain with an in-frame 57 bp deletion in the extracellular domain of Lepr. This suggests the deleted amino acids do not significantly affect Lepr structure and function. This is the first report of generating the Lepr mutant obese rat model in SD strain through a reverse genetic approach. This suggests that TALEN is an efficient and powerful gene editing technology for the generation of disease models.
\end{abstract}

TALENs, Lepr, knockout rat, germ-line transmission

Citation: Chen, Y., Lu, W., Gao, N., Long, Y., Shao, Y., Liu, M., Chen, H., Ye, S., Ma, X., Liu, M., and Li, D. (2017). Generation of obese rat model by transcription activator-like effector nucleases targeting the leptin receptor gene. Sci China Life Sci 60, 152-157. doi: 10.1007/s11427-016-5049-y

\section{INTRODUCTION}

Leptin receptor (Lepr) belongs to the super family of cytokine receptors which play key roles in maintaining body weight. It is a single-transmembrane domain protein with several alternatively spliced isoforms that are distributed in many types of tissues (Kielar et al., 1998; Tartaglia et al., 1995). Lepr deficiency is associated with obesity and obe-

$\dagger$ Contributed equally to this work

*Corresponding author (email: myliu@bio.ecnu.edu.cn)

***Corresponding author (email: dlli@bio.ecnu.edu.cn) sity-related diseases such as diabetes (Rosmond et al., 2000). The $\mathrm{db} / \mathrm{db}$ mouse strain carrying a point mutation leading to premature termination of the Lepr gene has been widely used as a model for obesity, diabetes and metabolic syndrome (Chen et al., 1996). The Zucker fatty rat (ZFR) strain also contains a Gln269Pro substitution in Lepr (Phillips et al., 1996) and the Koletsky rat (f) strain contains a T2349A transversion resulting in a Tyr763Stop nonsense mutation in Lepr just before the transmembrane domain (Wu-Peng et al., 1997). Because having an identical genetic background is critical for animal models in both basic scientific studies and innovative drug discovery, we sought to 
generate Lepr mutant rat strains through a reverse genetic approach.

Site-specific genetically modified rats have been generated by our group using zinc finger nucleases (ZFNs) (Geurts et al., 2009), gene-targeting in embryonic stem cells (Tong et al., 2010), transcription activator-like effector nucleases (TALENs) (Tesson et al., 2011), and most recently by the clustered regularly interspaced short palindromic repeats/Cas9 (CRISPR/Cas9) system (Li et al., 2013). Among the available technologies, although embryonic stem cell based gene targeting shows the best accuracy performance, it is a time- and labor-consuming strategy demanding great expense and experienced scientists, and it usually yields a low germline transmission efficiency (Tong et al., 2011). Through direct injection of ZFN pairs into one-cell embryos, a gene modified rat can be generated. However, optimization of zinc-finger modular arrays is also costly and off-target digestion is also reported (Urnov et al., 2010). Previously, we have shown that the CRISPR/Cas9 system is a powerful and highly efficient technology with less than ten days of hands-on working time (Shao et al., 2014). However, off-targeting excision induced a relatively high frequency of undesired mutations that are a major adverse effect (Pattanayak et al., 2013). The DNA binding domain of TALENs is derived from transcription activator-like effector (TALE) proteins which were originally discovered from the plant pathogenic bacteria Xanthomonas (Moscou and Bogdanove, 2009). TALEs possess a different single 34 aa module distinct from zinc finger motifs that recognizes the corresponding single target nucleotides having more predictable DNA-binding rules (Boch et al., 2009). Tesson and colleagues have reported the first establishment of a targeted-knockout rat by TALENs (Tesson et al., 2011). We have recently demonstrated that the TALENs are an effective genetic tool for rapid gene disruption with high efficiency and heritability in the mouse with distinct genetic background (Qiu et al., 2013).

Here we demonstrate the feasibility of generating Lepr KO rats via TALEN-mediated gene disruption. We successfully generated genetically modified Lepr rats in a SD genetic background that exhibited a high efficiency of germline transmission. We observed both a bi-allele mutant founder and three homozygous Lepr $\mathrm{KO}$ rat lines with distinct genomic mutations.

\section{RESULTS AND DISCUSSION}

\section{Design of TAL modules and rat Lepr locus targeting in vivo}

According to the rat Lepr nucleic acid sequence obtained from the NCBI (National Center for Biotechnology Information) genbank database, we designed a pair of TALE modular arrays targeting two adjacent sites with an $18 \mathrm{bp}$ interval (spacer) on exon 2 of the rat Lepr gene. The TALE arrays were fused to the $\mathrm{N}$ terminus of the catalytic domain of Fok I nuclease. In vitro transcribed mRNA of the TALEN pair was injected into 153 rat one-cell embryos and this resulted in $3 \mathrm{~F}_{0}$ newborns. Using the $\mathrm{T} 7$ endonuclease I (T7EI) digestion assay, we identified two $\mathrm{F}_{0}$ pups that were founders containing mutations in the Lepr locus. Interestingly, founder 2 rat carried a bi-allelic mutation and no wild-type PCR band was identified (Figure 1A). To determine the exact mutation sequence, PCR products were TA cloned and at least ten colonies were sequenced. All mutations of Lepr were identified and listed in Figure 1A. In summary, founder 1 had two mutants: a non-frame-shifting $57 \mathrm{bp}$ deletion and an $18 \mathrm{bp}$ insertion which generated a TGA premature stop codon. Since all ten TA clones from founder 2 rat exhibited a 394 bp deletion in the Lepr locus, this suggested that the founder was homozygous (Figure 1B). It was confirmed that founder 2 rat exhibited an obese phenotype by 18 weeks of age (Figure 1C). The mutation rate was high, as was previously reported (Tesson et al., 2011), supporting our studies in mouse embryos that the optimal spacer length is about 15-19 bp (Qiu et al., 2013).

\section{Highly efficient germline transmission of TALEN-induced Lepr mutations}

Successful germ-line transmission is important for the establishment of knockout rat lines. Two founders were separately backcrossed with WT SD rats to determine the germline transmission efficiency. Indeed, founder 2 rat showed infertility, further suggesting that it was homozygous. The germline transmission rate of founder 1 rat reached $100 \%$ as detected by the T7E1 assay (Figure 1D). To determine the mutations generated in individual founders, PCR products were sequenced (Figure 1E). In addititon to two mutant alleles being identified in the founder, a previously undetected allele with $1 \mathrm{bp}$ deletion was also determined in the $F_{1}$ pups. This suggests an incomplete sequencing of the TA clones or an uneven distribution of certain mutation-bearing cells between the toes and germ cells (Figure 1E). These results indicate that TALEN-induced gene mutation is highly heritable in rats.

\section{Characterization of the phenotypes of Lepr mutant rat}

Three strains of rats containing different Lepr mutations were generated separately by intercrossing between the $F_{1}$ generations with the same mutation. We named them Lepr $^{\Delta^{57 /} \Delta^{57}}$ rat (deletion $57 \mathrm{bp}$ ), Lepr ${ }^{\Delta^{1 / \Delta^{1}}}$ rat (deletion $1 \mathrm{bp}$ ) and Lepr $^{\text {in } 18 / \text { in } 18}$ rat (insertion $18 \mathrm{bp}$ ). The non-frame-shifting $57 \mathrm{bp}$ deletion resulted in a 19 amino acid deletion in the extracellular domain of Lepr in eepr $^{\Delta^{57 /} \Delta^{57}}$ rats. The Lepr $^{\Delta^{1 / \Delta^{1}}}$ strain contained a frame-shifting 1 bp deletion which disrupted gene function. An $18 \mathrm{bp}$ random sequence containing a TGA stop codon was inserted into the Lepr locus in Lepr $^{\text {in18/in18 }}$ strain which lead to premature termination of transcription. The earliest onset of obesity was observed at 5-6 weeks in $\operatorname{Lepr}^{\Delta^{1 / \Delta^{1}}}$ and Lepr $^{\text {in18/in18 }}$ rats, but not in $\operatorname{Lepr}^{\Delta}{ }^{57 /} \Delta^{57}$ rats. This indicated that the corresponding 


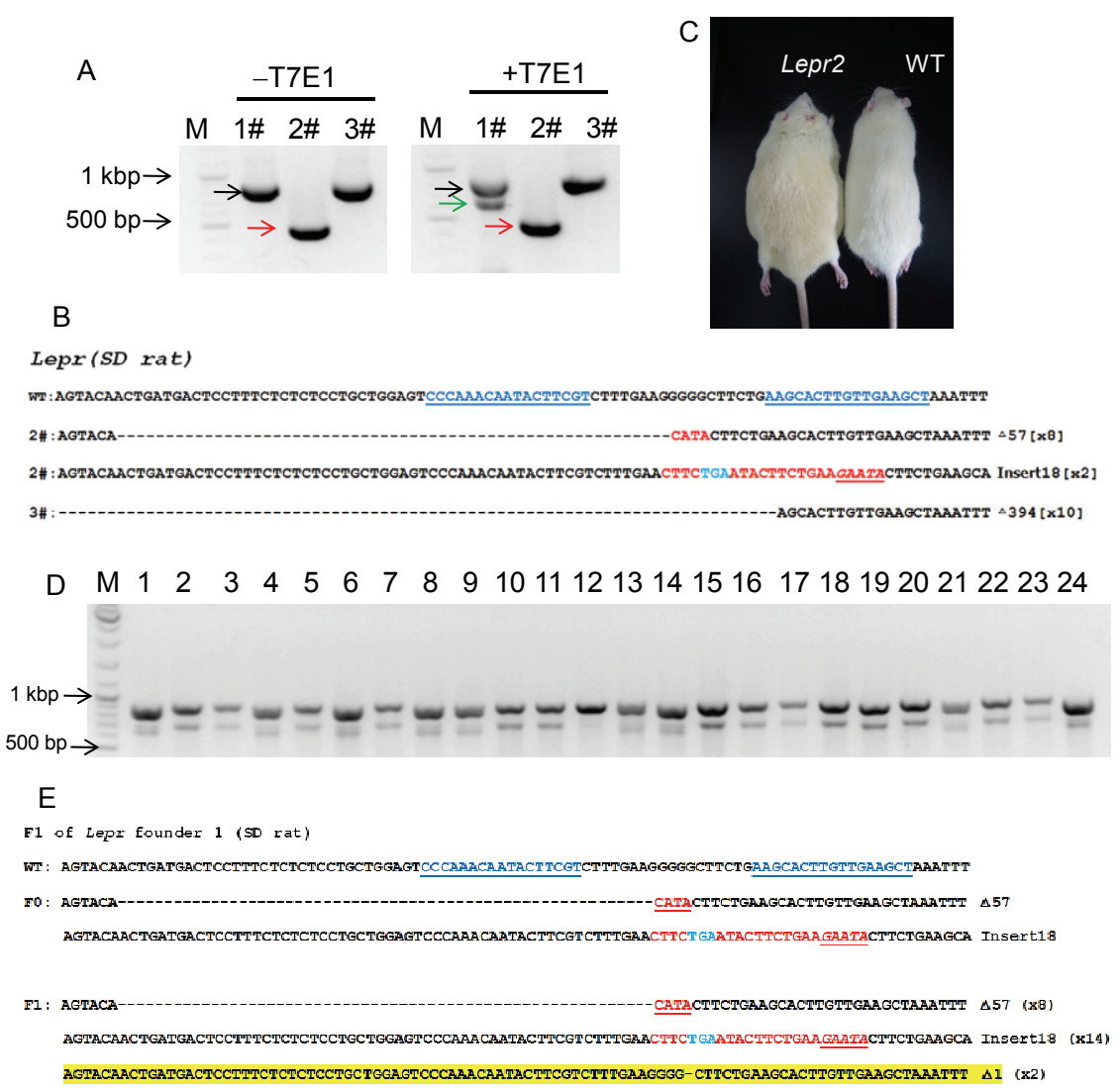

Figure 1 TALEN-induced mutations of Lepr in rat with efficient germline transmission. A, Detection of mutations in $\mathrm{F}_{0}$ rats generated by injection of TALEN mRNA targeting Lepr using T7 endonuclease assay. PCR primers were designed flanking the TALEN binding sites of Lepr. PCR products (WT, $871 \mathrm{bp}$ ) were melted, and annealed with a similar amount of PCR products amplified from wild-type mouse genomic DNA to form heteroduplex DNA. The DNA heteroduplex was then subjected to mismatch sensitive endonuclease (T7E1) assays and the products were resolved in 1.5\% agarose gels. Black arrow indicates wild-type rat, Green arrow indicates rat bearing mutations, red arrow indicates rat bearing bi-allelic mutation. M, DNA marker. B, Frequencies and sequences of TALEN-induced mutations in SD rats. The binding sites of the TALEN half-site are shown in blue and underlined. A dash represents a single base deletion. Insertions or substitutions are indicated in red letters. The length and frequency of indels are labeled to the right of each sequence. C, 18-week-old Lepr founder 2 (right) and its wild-type littermate (left) were anesthetized and photographed. D, Identification of $\mathrm{F}_{1}$ of Lepr founder 1 using T7 endonuclease assay. PCR primers were designed flanking the TALEN binding sites of Lepr. PCR products (871 bp) were melted, and annealed with a similar amount of PCR products amplified from wild-type mouse genomic DNA to form heteroduplex DNA. The DNA heteroduplex was then subjected to mismatch sensitive endonuclease (T7E1) assays and the products were resolved in 1.5\% agarose gels. Underlined data indicated rat bearing mutations. M, DNA marker. E, Sequences of TALEN-induced mutations in $\mathrm{F}_{1}$ of Lepr founder 1. The binding sites of the TALEN half-site are shown in blue and underlined. A dash represents a single base deletion. Insertions or substitutions are indicated by red letters and underlining. Allele that appeared in $F_{1}$ but was not originally identified in the founder is highlighted in yellow. The number of $F_{1}$ rats with each genotype is listed on the right. \#1, 3, 4, 8, 15, 18, 19, 20 of $F_{1}$ rats shown in (D) containing 57 bp deletion; \#2, 5, 6, 7, 10,11, 12, 13, 16, 17, 21, 22, 23, 24 of $\mathrm{F}_{1}$ rats containing 18 bp insertion; \#9,14 of $\mathrm{F}_{1}$ rats containing 1 bp deletion.

19 amino acids in the extracellular domain of Lepr were not indispensable for its function. Firstly, we detected the relative expression of Lepr in different tissues of Lepr ${ }^{\text {in 18/in18 }}$ and wild type littermates. We found that the expression of Lepr was significantly lower than that of wild type littermates with regard to brain, abdominal adipose, kidney, and small intestine (Figure 2A). This suggests that the premature stop codon of Lepr significantly reduced its mRNA stability in Lepr ${ }^{\text {in18/in18 }}$ rat, as it is well known that nonsense mutation induces mRNA decay (Hentze and Kulozik, 1999). Next, the body weight of Lepr $^{\text {in18/in18 }}$ and WT male rats were determined at 13 weeks of age (Figure 2B, Left). Lepr ${ }^{\text {in18/in18 }}$ rats were significantly heavier than wild type littermates (603.8 g, SE=7.77 vs. $366.5 \mathrm{~g}, \mathrm{SE}=12.32, P<0.0001)$. In the glucose tolerance test (IPGTT), Lepr ${ }^{\text {in } 18 / \text { in } 18}$ rats showed that the blood glucose levels were significantly higher than those of wild type littermates at all tested time points (Figure 2B, Middle). Using the insulin tolerance test (ITTs), Lepr ${ }^{\text {in18/in18 }}$ rats showed decreased sensitivity to injection of insulin than did wild type littermates, indicating resistance to insulin treatment (Figure 2B, Right). These data indicated that glucose homeostasis of Lepr $^{\text {in18/in18 }}$ rats were severely impaired. Moreover, examination of abdominal fat (ABT) from Lepr $^{\text {in18/in18 }}$ rats revealed a considerable cellular hypertrophy 

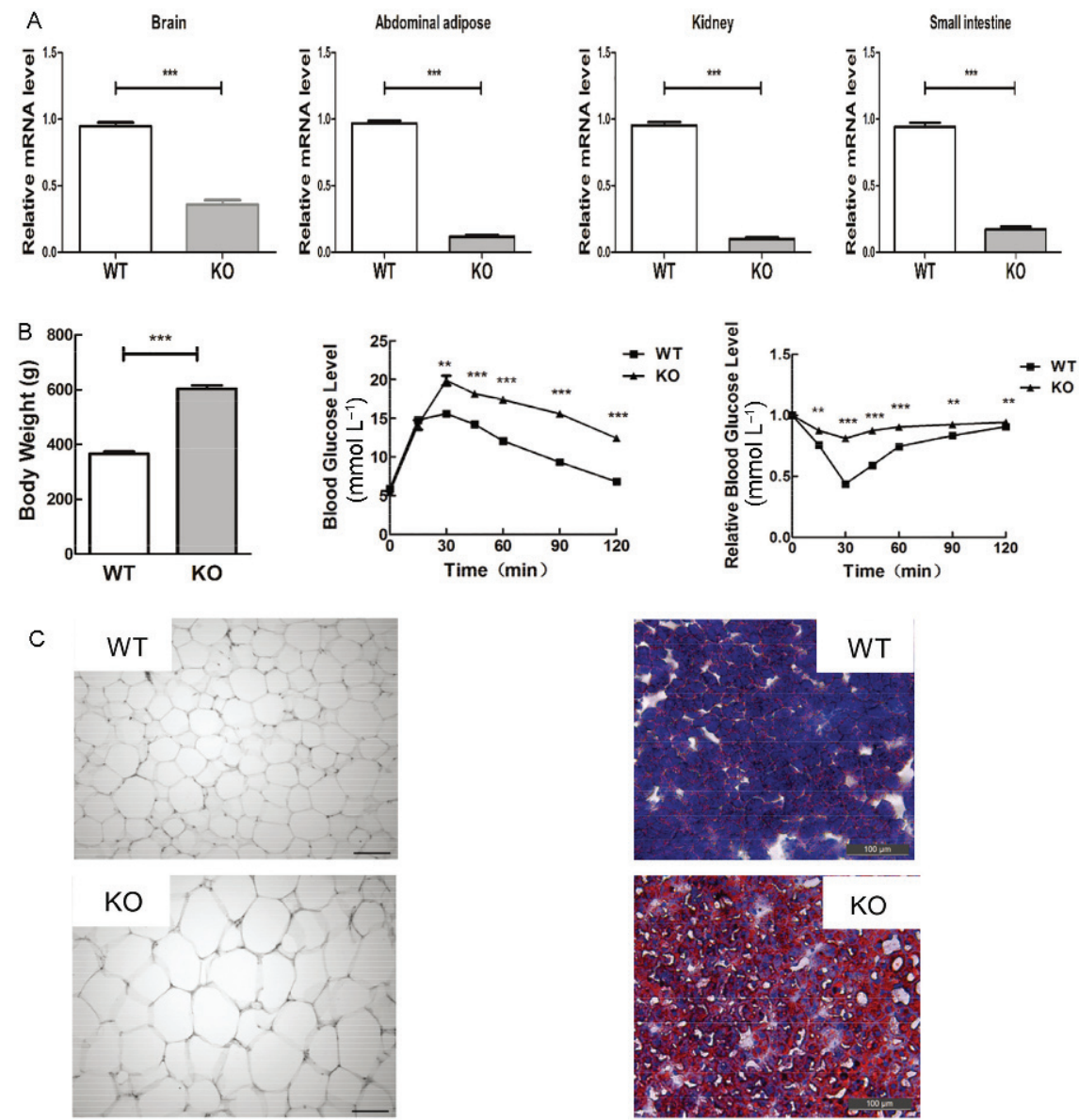

Figure 2 Phenotypes of Lepr ${ }^{\text {inl8/inls }}$ rats. A, Relative mRNA level of Lepr in variant tissues of Lepr ${ }^{\text {in } 18 / \text { in } 18}$ and wild type littermates. ***, P<0.001 by two-tailed $t$-test. B, Left, Body weight of 13 -week-old Lepr $^{\text {inl } 18 / i n 18}$ rats and its wild-type littermate. Middle, Lepr $r^{\text {in } 18 / i n 18}$ and its wild-type littermates were fasted overnight $16 \mathrm{~h}$, intraperitoneal injection with glucose $\left(2 \mathrm{~g} \mathrm{~kg}^{-1}\right.$ body weight $)$ and whole-blood glucose levels were monitored $2 \mathrm{~h}$. Single Asterisks indicate significant differences $(P<0.05)$, double Asterisks indicate $P<0.01$, and triple Asterisks indicate $P<0.001$ between lean and obese rats, the same below. Right, Lepr $^{\text {inl8/inls }}$ and its wild-type littermates were fasted for up to $4 \mathrm{~h}$, and intraperitoneal injection with insulin (1.5 $\left.\mathrm{U} \mathrm{kg}^{-1}\right)$ and whole-blood glucose levels were monitored 2 h. C, Left, Comparison of H\&E-stained abdominal adipose of Lepr $r^{\text {in } 18 \text { /in } 18}$ (lower) and its wild-type littermate (upper). Dramatic cellular hypertrophy is observed in abdominal fat. Scale bars: $100 \mu \mathrm{m}$. Right, Oil-Red-O staining of liver sections shows marked increase of lipid accumulation in Lepr $^{\text {in } 18 / \text { in }^{18}}$ rats (lower) relative to its wild-type littermates (upper). KO indicates the Lepr ${ }^{\text {in18/in18 }}$ rats, WT indicates wild-type littermates, A, B and C $n=5$ per group.

compared to wild type littermates (Figure 2C, Left). Further, Oil-Red-O staining of frozen sections from Lepr $r^{\text {in } 18 / \text { in } 18}$ rat livers showed strong signals indicating lipids, while the counterpart sections from the littermate controls were barely stained (Figure 2C, Right). These data indicate that Lepr $^{\text {in 18/in18 }}$ rats showed a marked increase of lipid accumulation in the liver. We also compared the serum biochemical indexes of Lepr $^{\text {in18/in18 }}$ rats with wild type littermates. The Lepr $^{\text {in } 18 / \text { in18 }}$ rats displayed significantly higher levels of triglyceride, total cholesterol, LDL-cholesterol, AST/ALT (Table 1). The $\operatorname{Lepr}^{\Delta^{1 / \Delta^{1}}}$ rats showed a similar phenotype as Lepr $^{\text {in18/in18 }}$ rats (data not shown). In conclusion, these results suggested that our Lepr KO rat in a pure SD genetic background generated by TALENs is a valuable animal model for obesity and obesity-related disease research.

In this article, we successfully generated Lepr $\mathrm{KO}$ rats by TALENs by targeting exon 2 of the Lepr gene in a pure SD genetic background. We observed a robust phenotype of Lepr-deficient rats. The Lepr ${ }^{\Delta^{1 /} \Delta^{1}}$ and $L e p r^{\text {in18/in18 }}$ rats with a single background developed obesity and obesity-related features, such as hyperlipemia, insulin-resistance, and hypertriglyceridemia, in the same manner as Zucker fatty rats. Since it is in a pure genetic background which will be better for basic scientific research and drug development than Zucker rats with mixed genetic background, our TALENgenerated model rat strains possess distinct advantages for obesity research.

\section{MATERIALS AND METHODS}

\section{TALEN expression vector construction}

We identified target sites of the Lepr using the online software (http://boglabx.plp.iastate.edu/TALENT/help.php) (Cer- 
Table 1 Blood metabolic parameters of the WT and Lepr $^{\text {in } 18 / \text { in } 18}$ rats at 13 weeks of age ${ }^{\text {a) }}$

\begin{tabular}{ccc}
\hline Indices & WT & Lepr in $^{\text {in } 18}$ rats \\
\hline Triglyceride $\left(\mathrm{mmol} \mathrm{L}^{-1}\right)$ & $0.608 \pm 0.081$ & $1.795 \pm 0.128$ \\
Total cholesterol $\left(\mathrm{mmol} \mathrm{L}^{-1}\right)$ & $0.745 \pm 0.025$ & $1.653 \pm 0.118$ \\
LDL-cholesterol $\left(\mathrm{mmol} \mathrm{L}^{-1}\right)$ & $<0.26$ & $0.340 \pm 0.027$ \\
HDL-cholesterol $\left(\mathrm{mmol} \mathrm{L}^{-1}\right)$ & $0.505 \pm 0.017$ & $0.535 \pm 0.037$ \\
AST $\left(\mathrm{U} \mathrm{L} \mathrm{L}^{-1}\right)$ & $50.750 \pm 2.869$ & $73.500 \pm 3.797$ \\
ALT $\left(\mathrm{U} \mathrm{L}^{-1}\right)$ & $26.250 \pm 2.175$ & $40.500 \pm 2.327$ \\
AST/ALT & $1.955 \pm 0.101$ & $2.356 \pm 0.0397$ \\
\hline
\end{tabular}

a) LDL, Low density lipoprotein. HDL, High density lipoprotein. AST, aspartate aminotransferase. ALT, alanine aminotransferase. Analytes were measured in serum and these data were tested in ADICON Clinical Laboratories. Data are given as means \pm SEM for surviving animals $(n=5$ for WT and Lepr $^{\text {in18/in18 }}$ rats, respectively).

mak et al., 2011). Nucleotide-recognizing TALE single-unit vectors and TALEN expression vectors were obtained from Bo Zhang (Peking University). We constructed TALE repeat arrays and TALE nuclease expression vectors according to the methods as described (Huang et al., 2011).

\section{Preparation of TALEN-coding mRNA in vitro}

To prepare mRNA for microinjection, we linearized the TALEN expression vector by Not I restriction enzyme digestion, followed by phenol/chloroform extraction and ethanol precipitation. Then, following the manufacturer's instructions, the linearized DNA was transcribed using Sp6 mMESSAGE mMACHINE Kit (Ambion, USA). We recovered mRNA by lithium chloride precipitation and resuspended mRNA in nuclease-free water.

\section{Microinjection of one-cell zygotes}

Superovulation of SD rat females (SLAC Shanghai) was obtained by mating with males of the same strain. One-cell stage embryos were obtained from the ampulla oviducts of the superovulated females. The embryos were washed and incubated in KSOM embryo culture medium (Millipore, USA) for $3-4 \mathrm{~h}$ at $37^{\circ} \mathrm{C}, 5 \% \mathrm{CO}_{2}$ before injection. The mRNA of TALEN pairs at a concentration of $20 \mathrm{ng} \mu \mathrm{L}^{-1}$ was injected into the cytoplasm of one-cell stage embryos using an Eppendorf TransferMan NK2 micromanipulator. Injected zygotes were transferred into pseudopregnant female rats immediately after injection. The rats were housed in standard cages in a specific pathogen-free facility on a 12 h light/dark cycle with ad libitum access to food and water. All animal experiments conformed to the regulations drafted by the Association for Assessment and Accreditation of Laboratory Animal Care in Shanghai and were approved by the East China Normal University Center for Animal Research.

\section{Genotyping}

Genomic DNA was extracted from rat toes or tail clips according to a standard DNA extraction procedure. PCR was performed with the following primers (Lepr-F: 5'-GAGAGCCTATGGGGTATTTCCTA-3', Lepr-R: 5'-TACTTACCTAGTTGGCGGAAAAC-3'). The PCR products were re-annealed to enable heteroduplex formation and then digested with T7 endonuclease I (New England Biotech, UK) in a $10 \mu \mathrm{L}$ reaction volume at $60 \mathrm{ng} \mu \mathrm{L}^{-1}$ concentration for $30 \mathrm{~min}$ at $45^{\circ} \mathrm{C}$. The mixture was then redissolved and analyzed on a $1.5 \%$ agarose gel. The exact sequences of founders were determined by DNA sequencing. PCR products were cloned using a TA cloning kit (TaKaRa, Japan) following the manufacturer's instructions. At least 10 colonies were picked from each transformation and then sequenced with RV-M primer.

\section{Total RNA extraction and Q-PCR}

The rats were sacrificed and indicated tissues were dissected for total RNA extraction with RNAiso Plus (TaKaRa) kit. Primer pair for qRT-PCR detection of rat Lepr was synthesized, Lepr-RT-F: 5'-TGCTTGTTGTGAAGCCCGAT-3', Lepr- RT-R: 5'-CGCTGTCTACCAGCAGAGAT-3'. qRTPCR was performed with SYBR Green (TaKaRa).

\section{Glucose tolerance tests (IPGTTs)}

After overnight $16 \mathrm{~h}$ fasting, the rats were weighed and injected intraperitoneally with glucose $\left(2 \mathrm{~g} \mathrm{~kg}^{-1}\right.$ body weight). Blood samples were collected from the tip of the tail immediately prior to and 15, 30, 45, 60, 90 and $120 \mathrm{~min}$ after injection. Blood glucose levels were measured at each time point using an ACCU-CHEK ${ }^{\circledR}$ Performa Nano (Roche, Switzerland).

\section{Insulin tolerance tests (ITTs)}

The rats were fasted for up to $4 \mathrm{~h}$, after which they were weighed and intraperitoneally injected with insulin (1.5 U $\mathrm{kg}^{-1}$ ). Blood samples were collected from the tip of the tail prior to and 15, 30, 45, 60, 90 and 120 min after injection. Blood glucose levels were measured at each time point using an ACCU-CHEK ${ }^{\circledR}$ Performa Nano.

\section{Oil-Red-O staining}

The livers of rats were fixed and frozen. $5 \mu \mathrm{m}$ liver frozen sections were sliced from the frozen tissues and rinsed in $\mathrm{ddH}_{2} \mathrm{O}$. For staining, sections were placed in Oil-Red-O solution $(0.5 \%$ Oil-Red-O in $60 \%$ isopropyl alcohol) for 
$10 \mathrm{~min}$. Then sections were rinsed in $60 \%$ isopropyl alcohol for $30 \mathrm{~s}$, followed with $\mathrm{ddH}_{2} \mathrm{O}$, and counterstained in hematoxylin for $3 \mathrm{~min}$. Finally, sections were rinsed under running water for $15 \mathrm{~min}$ and mounted with aqueous solution.

\section{Blood biochemical index determination}

After overnight fasting, the rats were bled from their eye sockets. Blood was stored at $4^{\circ} \mathrm{C}$ for $2 \mathrm{~h}$ and then centrifuged at $13400 \times g, 4^{\circ} \mathrm{C}$, for $5 \mathrm{~min}$. Biochemical parameters of the rat serum were measured in ADICON Clinical Laboratories (INC, Shanghai).

\section{Statistical analysis}

The results are presented as means \pm SEM. Statistical analysis was performed by a Mann-Whitney test using GraphPad Prism 5 software (GraphPad Software, USA). $P<0.05$ is considered statistically significant.

Compliance and ethics The author(s) declare that they have no conflict of interest.

Acknowledgements This work was supported by the State Key Development Programs of China (2012CB910400 to Mingyao Liu), the National Natural Science Foundation of China (31371455, 31171318 and 81330049), and the Science and Technology Commission of Shanghai Municipality (14140900300).

Boch, J., Scholze, H., Schornack, S., Landgraf, A., Hahn, S., Kay, S., Lahaye, T., Nickstadt, A., and Bonas, U. (2009). Breaking the code of DNA binding specificity of TAL-type III effectors. Science 326 , 1509-1512.

Cermak, T., Doyle, E.L., Christian, M., Wang, L., Zhang, Y., Schmidt, C., Baller, J.A., Somia, N.V., Bogdanove, A.J., and Voytas, D.F. (2011). Efficient design and assembly of custom TALEN and other TAL effector-based constructs for DNA targeting. Nucleic Acids Res 39, e82.

Chen, H., Charlat, O., Tartaglia, L.A., Woolf, E.A., Weng, X., Ellis, S.J., Lakey, N.D., Culpepper, J., Moore, K.J., Breitbart, R.E., Duyk, G.M., Tepper, R.I., and Morgenstern, J.P. (1996). Evidence that the diabetes gene encodes the leptin receptor: identification of a mutation in the leptin receptor gene in $\mathrm{db} / \mathrm{db}$ mice. Cell 84, 491-495.

Geurts, A.M., Cost, G.J., Freyvert, Y., Zeitler, B., Miller, J.C., Choi, V.M., Jenkins, S.S., Wood, A., Cui, X., Meng, X., Vincent, A., Lam, S., Michalkiewicz, M., Schilling, R., Foeckler, J., Kalloway, S., Weiler, H., Menoret, S., Anegon, I., Davis, G.D., Zhang, L., Rebar, E.J., Gregory, P.D., Urnov, F.D., Jacob, H.J., and Buelow, R. (2009). Knockout rats via embryo microinjection of zinc-finger nucleases. Science $325,433$.
Hentze, M.W., and Kulozik, A.E. (1999). A perfect message: RNA surveillance and nonsense-mediated decay. Cell 96, 307-310.

Huang, P., Xiao, A., Zhou, M., Zhu, Z., Lin, S., and Zhang, B. (2011). Heritable gene targeting in zebrafish using customized TALENs. Nat Biotechnol 29, 699-700.

Kielar, D., Clark, J.S., Ciechanowicz, A., Kurzawski, G., Sulikowski, T., and Naruszewicz, M. (1998). Leptin receptor isoforms expressed in human adipose tissue. Metabolism 47, 844-847.

Li, D., Qiu, Z., Shao, Y., Chen, Y., Guan, Y., Liu, M., Li, Y., Gao, N., Wang, L., Lu, X., Zhao, Y., and Liu, M. (2013). Heritable gene targeting in the mouse and rat using a CRISPR-Cas system. Nat Biotechnol 31, 681-683.

Moscou, M.J., and Bogdanove, A.J. (2009). A simple cipher governs DNA recognition by TAL effectors. Science 326, 1501.

Pattanayak, V., Lin, S., Guilinger, J.P., Ma, E., Doudna, J.A., and Liu, D.R. (2013). High-throughput profiling of off-target DNA cleavage reveals RNA-programmed Cas9 nuclease specificity. Nat Biotechnol 31, 839-843.

Phillips, M.S., Liu, Q., Hammond, H.A., Dugan, V., Hey, P.J., Caskey, C.J., and Hess, J.F. (1996). Leptin receptor missense mutation in the fatty Zucker rat. Nat Genet 13, 18-19.

Qiu, Z., Liu, M., Chen, Z., Shao, Y., Pan, H., Wei, G., Yu, C., Zhang, L., Li, X., Wang, P., Fan, H., Du, B., Liu, B., and Li, D. (2013). High-efficiency and heritable gene targeting in mouse by transcription activator-like effector nucleases. Nucleic Acids Res 41, e120.

Rosmond, R., Chagnon, Y.C., Holm, G., Chagnon, M., Perusse, L., Lindell, K., Carlsson, B., Bouchard, C., and Bjorntorp, P. (2000). Hypertension in obesity and the leptin receptor gene locus. J Clin Endocrinol Metab 85, 3126-3131.

Shao, Y., Guan, Y., Wang, L., Qiu, Z., Liu, M., Chen, Y., Wu, L., Li, Y., $\mathrm{Ma}, \mathrm{X}$. , and Li, D. (2014). CRISPR/Cas-mediated genome editing in the rat via direct injection of one-cell embryos. Nat Protoc 9, 2493-2512.

Tartaglia, L.A., Dembski, M., Weng, X., Deng, N., Culpepper, J., Devos, R., Richards, G.J., Campfield, L.A., Clark, F.T., Deeds, J., Muir, C., Sanker, S., Moriarty, A., Moore, K.J., Smutko, J.S., Mays, G.G., Wool, E.A., Monroe, C.A., and Tepper, R.I. (1995). Identification and expression cloning of a leptin receptor, OB-R. Cell 83, 1263-1271.

Tesson, L., Usal, C., Menoret, S., Leung, E., Niles, B.J., Remy, S., Santiago, Y., Vincent, A.I., Meng, X., Zhang, L., Gregory, P.D., Anegon, I., and Cost, G.J. (2011). Knockout rats generated by embryo microinjection of TALENs. Nat Biotechnol 29, 695-696.

Tong, C., Huang, G., Ashton, C., Li, P., and Ying, Q.L. (2011). Generating gene knockout rats by homologous recombination in embryonic stem cells. Nat Protoc 6, 827-844.

Tong, C., Li, P., Wu, N.L., Yan, Y., and Ying, Q.L. (2010). Production of p53 gene knockout rats by homologous recombination in embryonic stem cells. Nature 467, 211-213.

Urnov, F.D., Rebar, E.J., Holmes, M.C., Zhang, H.S., and Gregory, P.D. (2010). Genome editing with engineered zinc finger nucleases. Nat Rev Genet 11, 636-646.

Wu-Peng, X.S., Chua, S.C., Jr., Okada, N., Liu, S.M., Nicolson, M., and Leibel, R.L. (1997). Phenotype of the obese Koletsky ( $f$ ) rat due to Tyr763Stop mutation in the extracellular domain of the leptin receptor (Lepr): evidence for deficient plasma-to-CSF transport of leptin in both the Zucker and Koletsky obese rat. Diabetes 46, 513-518. 